\title{
Lanzoprazole promotes gastric carcinogenesis in rats with duodenogastric reflux
}

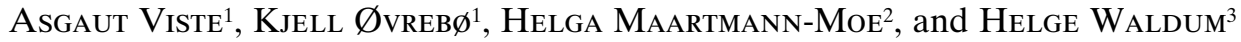 \\ ${ }^{1}$ Department of Surgery and The Gade Institute, Haukeland University Hospital, N-5021 Bergen, Norway \\ ${ }^{2}$ Department of Pathology, Haukeland University Hospital, Bergen, Norway \\ ${ }^{3}$ Department of Medicine, NTNU, Trondheim, Norway
}

\begin{abstract}
Background. Duodenogastric reflux is known to cause an increased frequency of cancer in the glandular portion of the stomach in rats. Furthermore, it is debated whether inhibition of gastric acid secretion may promote gastric carcinogenesis. In the present study we examined the combined effect of gastroduodenal reflux and acid inhibition with respect to the development of gastric carcinoma in the rat.

Methods. Following the construction of a gastrojejunostomy in male Wistar rats, half of them were given the proton pump inhibitor lanzoprazole for 1 year. The rats were then killed and the $\mathrm{pH}$ in the stomach and gastrin in blood were measured. The stomach was examined macroscopically as well as histologically.

Results. Gastrin levels at autopsy were significantly increased in treated rats compared to the control group, confirming an effect of lanzoprazole on gastric acid secretion. Body weight was significantly reduced in the treated rats. Thirty of 79 rats developed gastric cancer, and they were all adenocarcinomas of the Lauren intestinal type. Gastric cancers occurred significantly more often in lanzoprazole-treated rats $(50 \%)$ compared with controls $(27 \%)$.

Conclusion. Lanzoprazole given orally enhances the carcinogenic effect of duodenogastric reflux in rats.
\end{abstract}

Key words Carcinogenesis - Stomach - Carcinoma $\cdot$ Experimental $\cdot$ Rat $\cdot$ Proton pump inhibitors $\cdot$ Duodenogastric reflux

\section{Background}

The widespread use of proton pump inhibitors has led to concern about the consequences of profound acid suppression [1,2]. Potential areas of concern regarding the longterm use of proton pump inhibitors include carcinoid formation, development of gastric adenocarcinomas, bacterial overgrowth, and enteric infections.

Offprint requests to: A. Viste

Received: March 4, 2003 / Accepted: October 8, 2003
Pathological duodenogastric reflux is related to the development of gastritis and metaplasia, and possibly also gastroesophageal reflux disease [3]. Epidemiological cohort studies have revealed that there is an increased risk of gastric cancer following distal gastric resection [4,5], and it is likely that the main factor of this higher risk is duodenogastric reflux induced by surgery. Experimental studies have also shown that gastric cancer can be induced by creating duodenogastric reflux, without the use of carcinogens [6-8]. Some animal studies have further indicated that susceptibility to gastric cancer development may be increased when acid suppression is added to duodenogastric reflux $[9,10]$.

The purpose of this study was to examine whether the proton pump inhibitor lanzoprazole would increase the frequency of gastric cancer in rats subjected to duodenogastric reflux.

\section{Methods}

One hundred and twenty Wistar male rats, weighing $277 \mathrm{~g}$ (median), were used for the experiment. The animals arrived at the facility at least 10 days before the experiments, and were standardized according to the FELASA accreditation scheme (Federation of European Laboratory Animals Science Association, Utrecht, Netherlands) on arrival.

The animals received only tap water during the last $12 \mathrm{~h}$ before surgery. Anesthesia was induced by the spontaneous inhalation of $5 \%$ halothane added to a 50:50 blend of oxygen and nitrous oxide circulated within an anesthesia chamber for small animals.

The abdomen was opened in the midline and the stomach and duodenum were exposed, carefully avoiding damage to blood vessels and the vagal nerves. All animals had a standardized 8-mm gastrojejunostomy along the greater curvature in the corpus region, starting $2 \mathrm{~mm}$ distally to the border of the forestomach. The 
anastomoses were performed by inverting the mucosa with an extramucosal 6-0 running absorbable suture (Vicryl; Ethicon, Norderstedt, Germany). The abdominal wall was closed in layers with a running suture (4/0 Vicryl).

Forty-one animals died in the early postoperative period, and the remaining 79 rats were divided randomly into two groups, one treated with lanzoprazole in the food and the other serving as the control. Lanzoprazole was given from the time of operation to the end of the experiment at a dose of $20 \mathrm{mg} / \mathrm{kg} / \mathrm{per}$ day. Lanzoprazole capsules were opened and the granules were mixed in a mass with the regular food.

The rats in the control group were housed in groups of four in cages, and rats in the treatment group were housed one per cage. All rats were housed on aspen bedding at conditions of temperature, $22 \pm 0.5^{\circ} \mathrm{C}$ and $50 \pm 10 \%$ relative humidity. The light cycle was $12 \mathrm{~h} /$ $12 \mathrm{~h}$ with $1 \mathrm{~h}$ simulated sunrise and sunset. The rats had free access to water and were fed an RM1 expanded diet (Special Diets Services, Witham, UK).

Only animals surviving 38 weeks of observation were evaluated.

After 52 weeks, the animals were killed under general anesthesia by severing the inferior caval vein, and blood sampling was performed for gastrin measurement. The stomach was removed, opened along the greater curvature, and pinned flat onto a corkboard, examined for macroscopic tumor formation, and embedded in $4 \%$ formaldehyde for $48 \mathrm{~h}$. Samples from the stomach that contained the anastomosis were processed for paraffin wax embedding and sectioning, and were stained with hematoxylin and eosin.

Light microscopy and a structured examination scheme were used for the histological evaluation. Histological lesions were classified into the following groups: (1), normal mucosa; (2), cystic glandular proliferation; (3), dysplasia; and (4), carcinoma. Carcinoma of the mucosa was diagnosed by the identification of an irregular glandular growth pattern, cellular atypia, and invasiveness of the glandular structures in the mucosa or beyond the muscularis mucosa. Tumors that penetrated the muscularis mucosa were considered to be carcinomas. The histopathological examination was done by a pathologist (H.M.M.) who was unaware of whether or not the rat had received lanzoprazole.

The significance of differences between the groups was assessed by the $\chi^{2}$ test with Yates correction, and $t$ tests were applied for continuous variables. Probabilities of less than 0.05 were considered significant. The experimental Animal Board of the Norwegian Department of Agriculture approved the experiments.

\section{Results}

Forty-one rats died in the early postoperative period of up to 38 weeks, and 8 died between 38 and 52 weeks. Seventy-one rats survived until 52 weeks after the operation and were evaluated together with the 8 that died between 38 and 52 weeks. There were 38 animals in the lanzoprazole group and 41 in the control group that could be analyzed. Average body weight at the time the animals were killed was $460 \mathrm{~g}$ in the lanzoprazole group and $490 \mathrm{~g}$ in the control group.

The characteristics of the lanzoprazole group and the control group are given in Table 1 . We found no difference in the stomach $\mathrm{pH}$ between the two groups, probably because all rats were only allowed tap water the last day before they were killed. Gastrin levels at autopsy were increased significantly in treated rats, confirming an effect of lanzoprazole on gastric acid secretion. Body weight was significantly lower in the treated rats compared to the control group.

Histopathological changes in the gastric mucosa were mainly found in the anastomotic area. Normal mucosa was found in 9 rats, while dilatation and cysts were found in 14, and these were equally distributed among the two groups. Totally, 30 rats developed carcinomas (Table 2). In the lanzoprazole-treated group, 19 of the 38 animals $(50 \%)$ had gastric cancer that was verified by microscopic examination, compared to 11 of the 41 $(27 \%)$ in the control group $(P<0.05)$. There was a good correlation between the macroscopic presence of tumor and the histopathological diagnosis. Two animals had gastric cancer without macroscopic changes, and 4 animals had macroscopic changes without cancer (polyps, ulceration, and inflammation). The characteristic presentation of normal mucosa and cancer is shown in Figs. 1 and 2.

Table 1. Characteristics of 79 rats with induced duodenogastric reflux

\begin{tabular}{lccl}
\hline & $\begin{array}{c}\text { Lanzoprazole } \\
(n=38)\end{array}$ & $\begin{array}{c}\text { Controls } \\
(n=41)\end{array}$ & $P$ \\
\hline Weight (52 weeks) & $420 \mathrm{~g}$ & $508 \mathrm{~g}$ & 0.04 \\
pH & 2.5 & 3.5 & 0.76 \\
Gastrin level (pmol/1) & 48 & 28 & 0.016 \\
Cancer & 19 & 11 & 0.04 \\
\hline
\end{tabular}

Table 2. Histologic findings in rats subjected to duodenogastric reflux

\begin{tabular}{lccc}
\hline & $\begin{array}{c}\text { Cystic } \\
\text { dilatation }\end{array}$ & Atypia & Carcinomas \\
\hline Lanzoprazole $(n=38)$ & 28 & 23 & 19 \\
Controls $(n=41)$ & 26 & 20 & 11
\end{tabular}




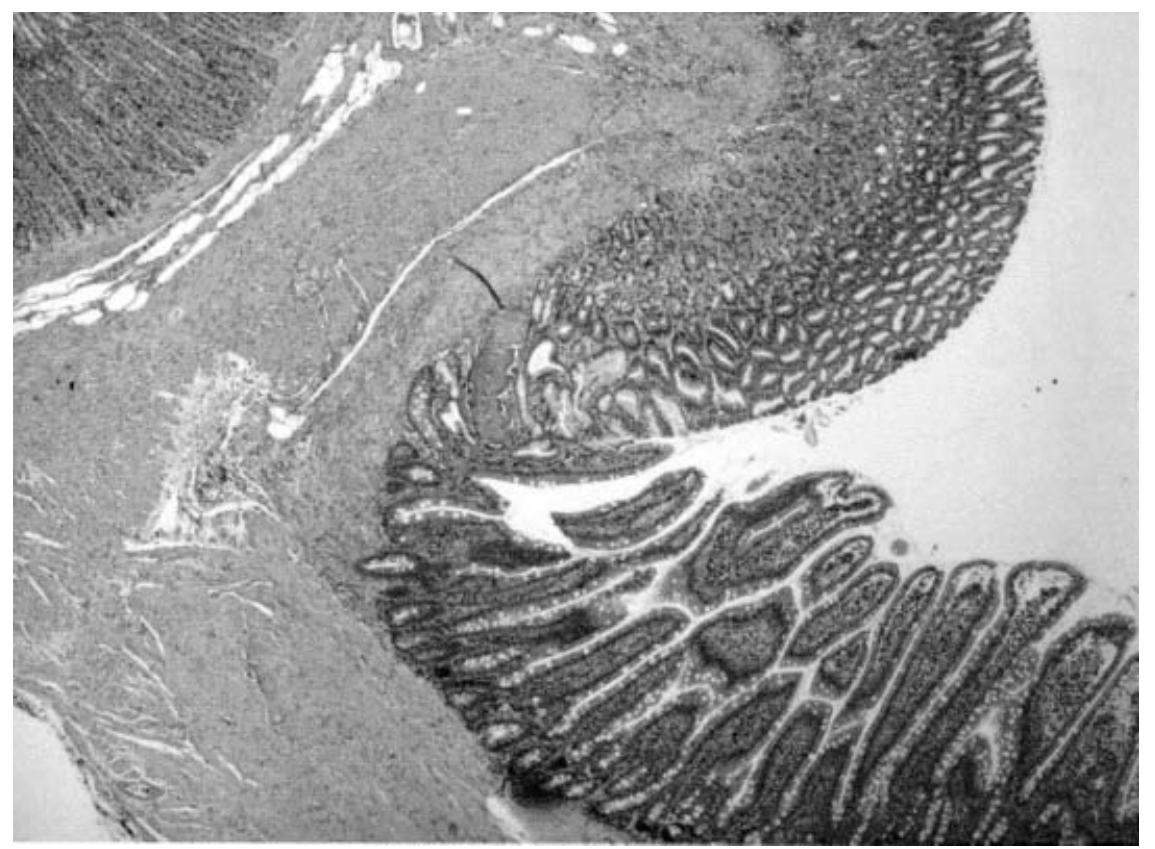

Fig. 1. Normal gastrojejunal anastomosis, showing a very distinct border between stomach and intestine. $\times 2.5$

\section{Discussion}

It is well known from experimental studies and epidemiological series that gastric cancer can be provoked by the establishment of duodenogastric reflux. The incidence is highest after gastrojejunostomy and a Billroth II resection, and lower after Billroth I resection [5]. Some authors have also shown that the incidence of malignant change in the gastric mucosa is proportional to the degree of duodenogastric reflux [7,11]. Wetscher et al. [3] found that duodenogastric reflux caused growth stimulation of the foregut mucosa and that this was potentiated by gastric acid blockade.

In our experiment, we observed a high peri- and postoperative mortality rate. Most published series do not state their postoperative mortality. Personal communication with several research groups has confirmed that mortality is a well-known problem when rats are used in an experimental setting, and it might reach a figure of $50 \%$.

In a previous study, we found no significant association between treatment with the $\mathrm{H}_{2}$-antagonist cimetidine and gastric cancer development in rats [9], although the study showed a tendency towards an increased cancer frequency in the cimetidine group. As we suspected that cimetidine was not providing profound acid suppression, the intention of the present study was to augment this effect by giving a potent proton pump inhibitor to the animals. Although the dosage of lanzoprazole given in the present study was 20 -fold that usually given to humans, the dose was of the same order as that given in comparable studies $[10,12-$ 14].

To our knowledge only one study has analyzed the combined effect of duodenogastric reflux and proton pump inhibition on gastric cancer development, and in that study the proton pump inhibitor omeprazole was used [10]. In the present study we found a twofold increase in gastric cancers in rats treated with the proton pump inhibitor lanzoprazole compared to the control group. Wetscher et al. [10] found a cancer frequency of $90 \%$ in their omeprazole group compared to $35 \%$ in the control group. In both these studies, most of the tumors were located in the anastomotic area, and, histologically, the tumors were highly differentiated adenocarcinomas.

There are several theories about how duodenogastric reflux may initiate gastric cancer. Gastric hypoacidity may enable nitrate-reducing bacteria to colonize the stomach and generate nitrites and potentially carcinogenic N-nitroso compounds. It has also been shown that a superimposed vagotomy can potentiate the carcinogenic effect of surgically established duodenogastric reflux [15]. The main reason for this is probably a reduction in acid output. Some studies have also shown that acid suppression with proton pump inhibitors might reduce vitamin B 12 uptake. However, no clinical impact of this effect has been noted over study periods of 3-5 years [16,17].

Another possibility is that the refluxate contains some until now unknown deleterious factors. Mason et al. [18] found no cancers following a pure bile reflux, 

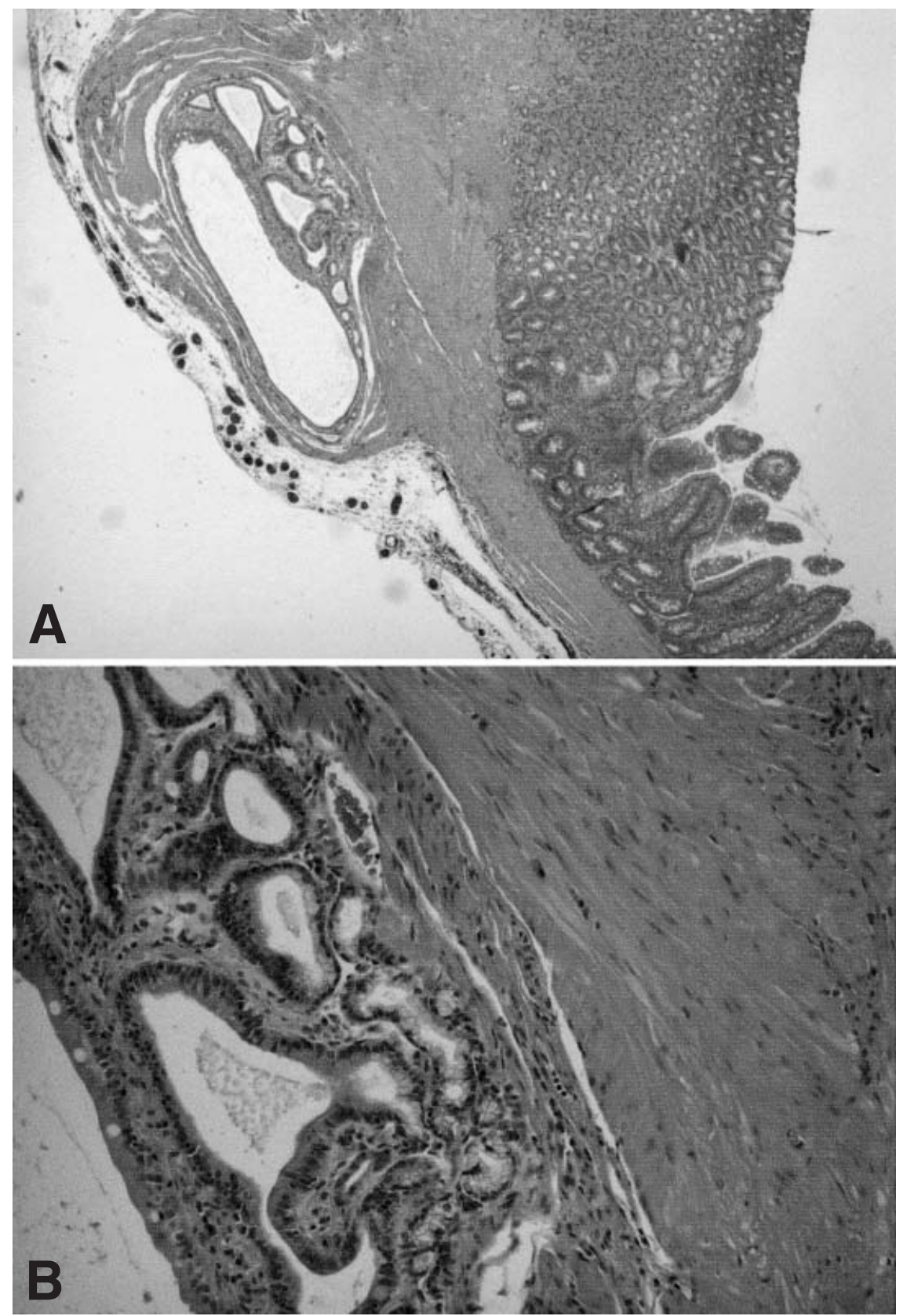

Fig. 2A,B. Highly differentiated adenocarcinoma infiltrating through the submucosa and into the muscularis propria. A $\times 2.5 ; \mathbf{B} \times 10$ versus carcinomas in 10 of 14 rats with pancreaticoduodenal reflux. However, Miwa et al. [19] showed that bile reflux caused a higher cancer frequency than pancreatic juice reflux.

It is well known that treatment with proton pump inhibitors will increase the gastrin level in the stomach and thereby stimulate growth of the oxyntic mucosa $[14,20]$. This effect is most probably exerted by histamine release from enterochromafin-like (ECL) cells. The ECL cell is assumed to be the main target cell of gastrin in the oxyntic mucosa. Thus, every condition with longterm hypergastrinemia tends to cause ECL- cell proliferation and, possibly, ECL-cell tumors. As for the effect of proton pump inhibitors, they are known to cause an increased rate of neuroendocrine gastric tumors, although the relation to adenocarcinomas is not completely clarified [21,22]. There is now some evidence showing that not only relatively benign ECL-cell carcinoids but also poorly differentiated neuroendocrine carcinomas originate from the ECL cell [23].

Proton pump inhibitors are generally believed to be safe drugs for human beings, and it is not clarified what implications findings in a rat model will have in other species. However, it can be concluded that there is sub- 
stantial evidence showing that the risk of gastric cancer development is increased in rats when treatment with proton pump inhibitors is superimposed on duodenogastric reflux.

Acknowledgments The study was supported by the Norwegian Cancer Society. The pharmacological agent lanzoprazole was generously provided by the company Wyeth-Lederle.

\section{References}

1. Waldum HL, Brenna E. Personal review: is profound acid inhibition safe? Aliment Pharmacol Ther 2000;14:15-22.

2. Laine L, Ahnen D, McClain C, Solacia E, Walsh JH. Potential gastrointestinal effects of long-term acid suppression with proton pump inhibitors. Aliment Pharmacol Ther 2000;14:651-68.

3. Wetscher GJ, Hinder RA, Kretchmar D, Stinson R, Perdikis G, Smyrk T, et al. Duodenogastric reflux causes growth stimulation of foregut mucosa potentiated by gastric acid blocade. Dig Dis Sci 1996;41:2166-73.

4. Viste A, Bjørnestad E, Opheim A, Skarstein A, Thunold J, Hartveit F, et al. Risk of carcinoma following gastric operations for benign disease: a historical cohort study of 3470 patients. Lancet 1996;II:502-4.

5. Lundegardh G, Adami HO, Hehnick C, Zack M, Meirik O. Stomach cancer after partial gastrectomy for benign ulcer disease. $\mathrm{N}$ Engl J Med 1988;319:195-200.

6. Miwa K, Hasegawa H, Fujimura T, Matsumoto H, Miyata R, Kosaka T, et al. Duodenal reflux through the pylorus induces gastric adenocarcinoma in the rat. Carcinogenesis 1992;13:2313-6.

7. Taylor PR, Mason RC, Filipe MI, Vaja S, Hanley DC, Murphy $\mathrm{GM}$, et al. Gastric carcinogenesis in the rat induced by duodenogastric reflux without carcinogens: morphology, mucin histochemistry, polyamine metabolism, and labelling index. Gut 1991;32:1447-54.

8. Seven R, Mercan S, Özarmagan S, Titiz I, Savci N, Dogan Ö. Nucleolar organizing regions in the operated rat stomach: relationship to metaplasia, dysplasia and carcinoma. Br J Surg 1993; 80:57-9.

9. Hortemo GS, Maartmann-Moe H, Røkke O, Viste A. Effect of cimetidine on development of gastric cancer in rats after gastrojejunostomy. Eur J Surg 1999;165:259-61.

10. Wetscher GJ, Hinder RA, Smyrk T, Perdikis G, Adrian TE, Profanter C. Gastric acid blockade with omeprazole promotes gastric carcinogenesis induced by duodenogastric reflux. Dig Dis Sci 1999;44:1132-5.

11. Schwab GP, Wetscher GJ, Klinger A, Kreczy A, Öfher C, Berresheim U, et al. Is there a dysplasia-carcinoma sequence in rat gastric remnant? Dig Dis Sci 1997;42:608-15.

12. Bolkent S, Yilmazer S, Kaya F, Ozturk M. Effects of acid inhibition on somatostatin-producing cells in the rat gastric fundus. Acta Histochem 2001;103:413-22.

13. Watanabe T, Higuchi K, Tominaga K, Fujiwara Y, Arakawa T. Acid regulates inflammatory response in a rat model of induction of gastric ulcer recurrence by interleukin-1 beta. Gut 2001;48:77481.

14. Lee H, Håkanson R, Karlsson A, Mattsson H, Sundler F. Lanzoprazole and omeprazole have similar effects on plasma gastrin levels, enterochromaffin-like cells, gastrin cells and somatostatin cells in the rat stomach. Digestion 1992;51:12532.

15. Kaminishi M, Shimizu N, Shiomoyama S, Yamaguchi H, Ogawa $\mathrm{T}$, Sakai S, et al. Etiology of gastric remnant cancer with special reference to the effects of denervation of the gastric mucosa. Cancer 1995;75:1490-6.

16. Koop H, Bachem MG. Serum iron, ferritin and vitamin $B_{12}$ during prolonged omeprazole therapy. J Clin Gastroenterol 1992;14:18892.

17. Marcuard SP, Albernaz L, Khazanie PG. Omeprazole therapy causes malabsorption of cyanocobalamin (vitamin $\mathrm{B}_{12}$ ). Ann Intern Med 1994;120:211-5.

18. Mason RC, Taylor PR, Filipe MI, McColl I. Pancreaticoduodenal secretions and the genesis of gastric stump carcinoma in the rat. Gut 1991;32:1447-54.

19. Miwa K, Fujimura T, Hasegawa H, Kosaka T, Miyata R, Miyazaki I, et al. Is bile or are pancreaticoduodenal secretions related to gastric carcinogenesis in rats with reflux through the pylorus? J Cancer Res Clin Oncol 1992;118:570-4.

20. Freston JW. Clinical significance of hypergastrinemia: relevance to gastrin monitoring during omeprazole therapy. Digestion 1992;51 (Suppl 1):102-14.

21. Haga Y, Nakatsura T, Shibata Y, Sameshima H, Nakamura Y, Tanimura M, et al. Human gastric carcinoid detected during longterm antiulcer therapy of $\mathrm{H}_{2}$ receptor antagonist and proton pump inhibitor. Dig Dis Sci 1998;43:253-7.

22. Mattsson H, Havu N, Bräutigam J, Carlsson K, Lundell L, Carlsson E. Partial gastric corpectomy results in hypergastrinemia and development of gastric enterochromaffin-like cell carcinoids in the rat. Gastroenterology 1991;100:311-19.

23. Rindi G, Azzoni C, La Rosa S, Klersy C, Paolotti D, Rappel S, et al. ECL tumors and poorly differentiated endocrine carcinoma of the stomach: prognostic evaluation by pathological analysis. Gastroenterology 1999;116:532-42. 